CAHIERS DE

NARRATOLOGIE
Cahiers de Narratologie

Analyse et théorie narratives

$33 \mid 2018$

L'Art du roman chez Umberto Eco

\title{
Sue J. Kim, On Anger : Race, Cognition, Narrative
}

Julien Quesne

\section{(2) OpenEdition}

\section{Journals}

Electronic version

URL: https://journals.openedition.org/narratologie/8488

DOI: $10.4000 /$ narratologie. 8488

ISSN: 1765-307X

\section{Publisher}

LIRCES

\section{Electronic reference}

Julien Quesne, "Sue J. Kim, On Anger : Race, Cognition, Narrative", Cahiers de Narratologie [Online], 33 |

2018, Online since 23 July 2018, connection on 21 September 2021. URL: http://

journals.openedition.org/narratologie/8488 ; DOI: https://doi.org/10.4000/narratologie.8488

This text was automatically generated on 21 September 2021

Article L.111-1 du Code de la propriété intellectuelle. 


\title{
Sue J. Kim, On Anger : Race, Cognition, Narrative
}

\author{
Julien Quesne
}

\section{REFERENCES}

Sue J. Kim, On Anger : Race, Cognition, Narrative, University of Texas Press, Austin, 2013

\section{AUTHOR'S NOTE}

*Toutes les citations de l'ouvrage ont fait l'objet d'une traduction de l'auteur.

1 Le choix d'effectuer le compte rendu d'un ouvrage écrit en langue anglaise s'est imposé avant tout de par l'originalité du travail de Sue J. Kim dans son étude pluridimensionnelle de la colère : la diversité des approches abordées confère à cet ouvrage une profondeur heuristique indéniable. Plus globalement et dans un contexte scientifique international où l'anglais maintient son hégémonie dans la plupart des disciplines, l'accès à ce genre d'ouvrage peut être décisif pour contrer l'appauvrissement des échanges sur ce type de questions avec le monde nonanglophone.

2 À travers cet ouvrage, Sue J. Kim met en évidence la nécessité d'une ouverture à d'autres disciplines pour saisir son objet, comme pour mieux sortir des approches concevant les émotions dans un cadre d'interprétation strictement neurocognitif ou misant sur l'antagonisme avec les sciences sociales. Dans les chapitres 1 et 2, l'auteure discute de la colère raciale, ou plutôt racialisée, à l'intersection des études cognitives et culturelles, arguant qu'une compréhension dialectique de ces deux dimensions est incontournable pour saisir ce phénomène émotionnel. Sur le registre des émotions, l'auteure insiste sur le fait que la colère s'inscrit au sein d'un rapport dialectique (6). Les chapitres 3, 5, 6 et 7 sont basés sur l'étude de plusieurs narratifs (romans, films, séries télé) afin de démontrer en quoi la race est une dimension centrale pour expliquer 
les représentations et les expériences liées à la colère. Pour paraphraser Kim : personne n'est en colère pour rien, cette dernière est toujours dirigée vers quelque chose ou quelqu'un (4). Plus particulièrement, l'existence d'une colère racialisée oblige l'auteure à s'interroger sur les fondements historiques, coloniaux et racistes de son émergence.

D’une manière générale, l'auteure conçoit la colère comme un «système élastique qui ne peut se comprendre complètement qu'à travers une pluralité de dimensions: cognitive, affective, idéologique et matérialiste » (4)*. Cette approche analytique qui fait front à une perception cognitiviste essentiellement centrée sur l'individu s'appuie sur l'argument que la colère est tout à la fois une condition et une expérience située par la race, le genre et la classe. C'est contre une certaine vision réductionniste des émotions que l'auteure entend affirmer l'importance du rôle de l'histoire, du politique et des narratifs dans la construction sociale, culturelle et cognitive de cette émotion.

D'un côté, Kim reconnaît l'apport des études cognitives dans l'analyse des sources émotives et psychologiques de la colère, et particulièrement dans la théorie narrative cognitive. Elle fait toutefois le constat que ces études ont trop souvent porté sur les populations blanches occidentales privilégiées (9). En s'appuyant sur ces résultats, une partie des sciences cognitives a renforcé l'idée que la colère est une expérience strictement individuelle au sein de laquelle les catégories de race, de genre et de classe n'ont quasiment jamais été prises en compte. À ce titre, Kim met l'emphase sur les limites épistémologiques et méthodologiques de l'appraisal theory ( «la théorie de l'évaluation soutient que l'émotion est produite par la perception du succès ou de l'échec d'un sujet dans la réalisation d'un but désiré », 17), puisque cette vision instrumentale des émotions tendrait à rendre invisible le rôle de l'histoire coloniale et de la culture dans le déclenchement des émotions.

En conclusion du chapitre 1, Kim insiste pour que les sciences sociales participent à la révolution cognitive en cours, principalement afin que les analyses des émotions qui s'inscrivent dans des logiques cognitives au cœur de la relation sociale ne se situent plus dans l'angle mort des sciences sociales. Considéré que la cognition, par l'intermédiaire des émotions, est façonnée par la culture (29) et notamment les narratifs fictionnels, c'est participer à une remise en question épistémologique et politique d'une naturalisation de la colère racialisée. L'apprentissage par association a lié la colère à des pensées et à des images mentales en un réseau dense d'allers et retours, et à ce titre les niveaux cognitifs et émotionnels de traitement, tout comme les niveaux individuels et collectifs (32), sont toujours liés. Ces représentations de la colère sont fournies en grande partie par la fiction.

6 De l'autre côté, l'auteure s'appuie sur les études culturelles et le concept de "structure de sentiment» (60) de Raymond Williams, afin d'analyser avant tout la colère comme un phénomène politique et historique. S'appuyant sur les théoriciennes féministes noires, Kim mentionne que «la revendication de la colère comme outil politique a refait surface, un peu ironiquement, en parallèle à la contestation de la pathologisation des corps racisés décrits comme irrationnels, pathologiquement en colère " (49). Toujours à partir des travaux de Patricia Hill Collins, Audre Lorde ou encore bell hooks, l'auteure défend l'idée de la rage noire comme celle d'un avantage épistémique fondée sur la communalité de l'expérience et permettant de saisir comment « cette colère est fondée sur le genre, la race, le sexe, la classe » (68).

7 Ces fondations d'une idée de la colère pathologique reposent en grande partie, écrit Kim, sur les représentations qui en sont données dans les productions culturelles. 
Comme le savent si bien les écrivains et écrivaines, les réalisateurs et réalisatrices, les romans et les films peuvent déclencher des réactions émotionnelles spontanées, somatiques et directes - parfois même de manière inattendue, involontaire et inconsciente. Ainsi Paul Haggis joue-t-il avec ces registres dans Crash [Collision, 2004], avec « une technologie des affects qui produit divers types de colère, en s'appuyant sur les outils de la théorie narrative » (67). Le film fait émerger, nous dit l'auteure, des représentations de la colère à travers les lunettes moralisatrices du multiculturalisme néolibéral qui considère à la fois l'individu comme le problème et la solution au racisme et sexisme. De même le chapitre 7, à travers l'analyse de la série The Wire de David Simon [2002-2008], "examine le social, l'économique, le politique, et autres forces institutionnelles qui produisent le phénomène de la colère, urbaine, des jeunes des minorités » (6).

8 Kim montre dans cet ouvrage en quoi les schémas narratifs présents dans les romans, films et séries télé étudiés, sont «la clé de voûte du conditionnement du sujet, du genre, du sexe, de la race, de la nationalité, et autres formations idéologiques, et de ce fait, façonnent de manière décisive les émotions » (6). À ce titre, l'auteure situe l'analyse de la colère à la jonction des études cognitives et culturelles « pour arriver à la conclusion que les émotions (et la cognition) sont inséparables des narratifs et de leurs contextes » (6).

\section{INDEX}

Mots-clés: émotions, cognition, narration, colère, sociologie, féminisme du point de vue situé, études culturelles

\section{AUTHOR}

\section{JULIEN QUESNE}

Doctorant en sociologie, Université du Québec à Montréal, quesne.julien_thomas@courrier.uqam.ca 